\title{
CLASSIFICATION OF LATTICE POLYTOPES WITH SMALL VOLUMES
}

\author{
TAKAYUKI HIBI AND AKIYOSHI TSUCHIYA
}

\begin{abstract}
In the frame of a classification of general square systems of polynomial equations solvable by radicals, Esterov and Gusev succeeded in classifying all spanning lattice polytopes whose normalized volumes are at most 4 . In the present paper, we complete to classify all lattice polytopes whose normalized volumes are at most 4 based on the known classification of their $\delta$-polynomials.
\end{abstract}

\section{INTRODUCTION}

One of the most important, however, unreachable goals of the study on lattice polytopes is to classify all of the lattice polytopes, up to unimodular equivalence. In lower dimension, the following classes of lattice polytopes are classified:

- 3-dimensional lattice polytopes with at most 11 lattice points $[8,9$, 10];

- 3-dimensional lattice polytopes with one interior lattice point [21];

- 3-dimensional lattice polytopes with two interior lattice points [1].

On the other hand, for arbitrary dimension, in each of the following classes of lattice polytopes, a complete classification is known:

- Centrally symmetric smooth Fano polytopes [26];

- Pseudo-symmetric smooth Fano polytopes [13, 26];

- Lattice polytopes with $\delta$-binomials $[3,4,6]$;

- Lattice polytopes with palindromic $\delta$-trinomials $[5,19]$.

It is fashionable among the study on lattice polytopes to classify the lattice polytopes with a given $\delta$-polynomial. In the present paper, we will classify all lattice polytopes of arbitrary dimension whose normalized volumes are at most 4 based on the known classification of their $\delta$-polynomials [16, 17]. In the frame of a classification of general square systems of polynomial equations solvable by radicals, Esterov and Gusev [12] succeeded in classifying all lattice polytopes $\mathcal{P} \subset \mathbb{R}^{d}$ whose normalized volumes are at most 4 for which $\mathbb{Z}\left((\mathcal{P}, 1) \cap \mathbb{Z}^{d+1}\right)=\mathbb{Z}^{d+1}$. (Here $\mathbb{Z}\left((\mathcal{P}, 1) \cap \mathbb{Z}^{d+1}\right)=\left\{z_{1} \mathbf{x}_{1}+\cdots+z_{n} \mathbf{x}_{n}\right.$ :

2010 Mathematics Subject Classification. 52B12, 52B20.

Key words and phrases. lattice polytope, $\delta$-polynomial, $\delta$-vector, Ehrhart polynomial, unimodular equivalence. 
$\left.z_{1}, \ldots, z_{n} \in \mathbb{Z}\right\}$ for $(\mathcal{P}, 1) \cap \mathbb{Z}^{d+1}=\left\{\mathbf{x}_{1}, \ldots, \mathbf{x}_{n}\right\} \subset \mathbb{Z}^{d+1}$.) However, the condition $\mathbb{Z}\left((\mathcal{P}, 1) \cap \mathbb{Z}^{d+1}\right)=\mathbb{Z}^{d+1}$ is rather strong for achieving a classification of lattice polytopes. For example, no empty simplex satisfies the property and, in addition, there exists a lattice non-simplex whose normalized volume is 4 and that lacks the property. Combining our work with a result of Esterov and Gusev [12] will establish a complete classification of lattice polytopes whose normalized volumes are at most 4 with their $\delta$-polynomials.

1.1. Possible $\delta$-polynomials. We recall a complete characterization of the $\delta$-polynomials of lattice polytopes whose normalized volumes are at most 4 .

Let us recall from [7] and [14, Part II] what the $\delta$-polynomial of a lattice polytope is. A lattice polytope is a convex polytope all of whose vertices have integer coordinates. Let $\mathcal{P} \subset \mathbb{R}^{d}$ be a lattice polytope of dimension $d$ and define $\delta(\mathcal{P}, t)$ by the formula

$$
\delta(\mathcal{P}, t)=(1-t)^{d+1}\left[1+\sum_{n=1}^{\infty}\left|n \mathcal{P} \cap \mathbb{Z}^{d}\right| t^{n}\right],
$$

where $n \mathcal{P}=\{n \mathbf{a}: \mathbf{a} \in \mathcal{P}\}$, the dilated polytopes of $\mathcal{P}$. Then it is known that $\delta(\mathcal{P}, t)$ is a polynomial in $t$ of degree at most $d$. We say that the polynomial $\delta(\mathcal{P}, t)=\delta_{0}+\delta_{1} t+\cdots+\delta_{d} t^{d}$ is the $\delta$-polynomial (or the $h^{*}$-polynomial) of $\mathcal{P}$ and the sequence $\delta(\mathcal{P})=\left(\delta_{0}, \delta_{1}, \ldots, \delta_{d}\right)$ is the $\delta$-vector (or the $h^{*}$-vector) of $\mathcal{P}$. The following properties of $\delta(\mathcal{P})$ are known:

- $\delta_{0}=1, \delta_{1}=\left|\mathcal{P} \cap \mathbb{Z}^{d}\right|-(d+1)$ and $\delta_{d}=\left|\operatorname{int}(\mathcal{P}) \cap \mathbb{Z}^{d}\right|$, where $\operatorname{int}(\mathcal{P})$ is the interior of $\mathcal{P}$. Hence one has $\delta_{1} \geq \delta_{d}$;

- $\delta_{i} \geq 0$ for each $i[23]$;

- When $\delta_{d} \neq 0$, one has $\delta_{i} \geq \delta_{1}$ for $1 \leq i \leq d-1[15]$;

- $\sum_{i=0}^{d} \delta_{i} / d$ ! coincides with the usual volume of $\mathcal{P}[24$, Proposition 4.6.30]. In general, the positive integer $\sum_{i=0}^{d} \delta_{i}$ is said to be the normalized volume of $\mathcal{P}$, denoted by $\operatorname{Vol}(\mathcal{P})$.

In [16, Theorem 5.1] and [17, Theorem 0.1], the possible $\delta$-polynomials with $\delta_{0}+\cdots+\delta_{d} \leq 4$ are completely classified.

Theorem 1.1 ([16, 17]). Let $2 \leq V \leq 4$ be a positive integer and $1+t^{i_{1}}+$ $\cdots+t^{i_{V-1}}$ a polynomial with $1 \leq i_{1} \leq \cdots \leq i_{V-1} \leq d$. Then there exists a lattice polytope of dimension $d$ whose $\delta$-polynomial equals $1+t^{i_{1}}+\cdots+t^{i_{V-1}}$ if and only if one of the following is satisfied:

(1) $V=2$ and $i_{1} \leq\lfloor(d+1) / 2\rfloor$;

(2) $V=3,2 i_{1} \geq i_{2}$ and $i_{1}+i_{2} \leq d+1$;

(3) $V=4, i_{3} \leq i_{1}+i_{2}, i_{1}+i_{3} \leq d+1$ and $i_{2} \leq\lfloor(d+1) / 2\rfloor$, and the additional condition

$$
2 i_{2} \leq i_{1}+i_{3} \text { or } i_{2}+i_{3} \leq d+1 \text {. }
$$


We remark that when $\delta_{0}+\cdots+\delta_{d} \leq 4$, all the possible $\delta$-polynomials can be obtained by lattice simplices. However, when $\delta_{0}+\cdots+\delta_{d}=5$, this is not true [16, Remark 5.3]. Most recently, the possible $\delta$-polynomials with $\delta_{0}+\cdots+\delta_{d}=5$ are completely classified $[18,25]$.

1.2. Main results. Recall that a matrix $A \in \mathbb{Z}^{d \times d}$ is unimodular if $\operatorname{det}(A)=$ \pm 1 . Given lattice polytopes $\mathcal{P}$ and $\mathcal{Q}$ in $\mathbb{R}^{d}$ of dimension $d$, we say that $\mathcal{P}$ and $\mathcal{Q}$ are unimodularly equivalent if there exist a unimodular matrix $U \in \mathbb{Z}^{d \times d}$ and a lattice point $\mathbf{w}$, such that $\mathcal{Q}=f_{U}(\mathcal{P})+\mathbf{w}$, where $f_{U}$ is the linear transformation in $\mathbb{R}^{d}$ defined by $U$, i.e., $f_{U}(\mathbf{v})=\mathbf{v} U$ for all $\mathbf{v} \in \mathbb{R}^{d}$.

For a lattice polytope $\mathcal{P} \subset \mathbb{R}^{d}$ of dimension $d$, the lattice pyramid over $\mathcal{P}$ is defined by $\operatorname{conv}(\mathcal{P} \times\{0\},(0, \ldots, 0,1)) \subset \mathbb{R}^{d+1}$. Let $\operatorname{Pyr}(\mathcal{P})$ denote this polytope. We often use the term lattice pyramid for a lattice polytope that has been obtained by successively taking lattice pyramids. Note that the $\delta$-polynomial does not change under lattice pyramids [2]. Therefore, it is essential that we classify lattice polytopes which are not lattice pyramids over any lower-dimensional lattice polytope.

A lattice polytope $\mathcal{P} \subset \mathbb{R}^{d}$ for which $\mathbb{Z}\left((\mathcal{P}, 1) \cap \mathbb{Z}^{d+1}\right)=\mathbb{Z}^{d+1}$ is called spanning. From the work of Hofscheier, Katthän and Nill it follows that there are only finitely many lattice spanning polytopes $\mathcal{P} \subset \mathbb{R}^{d}$ of given normalized volume (and arbitrary dimension) up to unimodular equivalence and lattice pyramid constructions [20, Corollary 2.4]. In particular, Esterov and Gusev [12] gave only finitely many lattice spanning polytopes whose normalized volumes are at most 4 for their classification result. However, it is hard to classify lattice non-spanning polytopes. In fact, there exist infinitely many lattice non-spanning polytopes of given normalized volume even up to unimodular equivalence and lattice pyramid constructions.

In the present paper, we will complete to classify, up to unimodular equivalence and lattice pyramid constructions, the lattice polytopes whose normalized volumes are at most 4 . The complete classification of the lattice polytopes whose normalized volumes are at most 4 up to unimodular equivalence consists of these polytopes and lattice pyramids over them. Note that every lattice simplex of dimension $d$ with $\operatorname{Vol}(\mathcal{P})=1$ is unimodularly equivalent to the standard simplex of dimension $d$. And the normalized volume of a lattice non-spanning non-simplex is at least 4. In order to classify all lattice polytopes whose normalized volumes are at most 4 , we should consider the following three cases:

(1) Lattice simplices $\Delta \subset \mathbb{R}^{d}$ with $\operatorname{Vol}(\Delta) \leq 4$;

(2) Lattice spanning non-simplices $\mathcal{P} \subset \mathbb{R}^{d}$ with $\operatorname{Vol}(\mathcal{P}) \leq 4$;

(3) Lattice non-spanning non-simplices $\mathcal{P} \subset \mathbb{R}^{d}$ with $\operatorname{Vol}(\mathcal{P})=4$.

The complete classification of the case (2) can be obtained from [12]. Therefore, we will show the cases (1) and (3).

Let $\mathbf{0}$ denote the origin of $\mathbb{R}^{d}$ and let $\mathbf{e}_{1}, \ldots, \mathbf{e}_{d}$ denote the canonical unit coordinate vectors of $\mathbb{R}^{d}$. First, the complete classification of the lattice simplices $\Delta \subset \mathbb{R}^{d}$ with $\operatorname{Vol}(\Delta) \leq 4$ can be obtained from the following: 
Theorem 1.2. Let $\Delta \subset \mathbb{R}^{d}$ be a lattice simplex of dimension $d$ whose $\delta$ polynomial equals $1+t^{i_{1}}+\cdots+t^{i_{V-1}}$ with $2 \leq V \leq 4$ and $1 \leq i_{1} \leq \cdots \leq i_{V-1}$. Assume that $\Delta$ is not a lattice pyramid. Then there exist, up to unimodular equivalence, exactly the following 5 possibilities for $\Delta$ :

(1) $V=2: \Delta^{(2)}$;

(2) $V=3: \Delta^{(3)}$;

(3) $V=4: \Delta_{i}^{(4)}, 1 \leq i \leq 3$.

The conditions and vertices of $\Delta$ are presented in TABLE 1.

\begin{tabular}{|c|c|c|}
\hline & conditions & vertices \\
\hline$\Delta^{(2)}$ & $2 i_{1}=d+1$ & $\mathbf{0}, \mathbf{e}_{1}, \ldots, \mathbf{e}_{d-1}, \mathbf{e}_{1}+\cdots+\mathbf{e}_{d-1}+2 \mathbf{e}_{d}$ \\
\hline$\Delta^{(3)}$ & $\begin{array}{l}2 i_{1} \geq i_{2} \\
i_{1}+i_{2}=d+1\end{array}$ & $\begin{array}{l}\mathbf{0}, \mathbf{e}_{1}, \ldots, \mathbf{e}_{d-1} \\
2 \sum_{i=1, i \neq d}^{-i_{1}+2 i_{2}-1} \mathbf{e}_{i}+\sum_{i=-i_{1}+2 i_{2}}^{d-1} \mathbf{e}_{i}+3 \mathbf{e}_{d}\end{array}$ \\
\hline$\Delta_{1}^{(4)}$ & $\begin{array}{l}i_{1}<i_{2}<i_{3} \\
i_{3} \leq i_{1}+i_{2} \\
2 i_{2}=i_{1}+i_{3}=d+1\end{array}$ & $\begin{array}{l}\mathbf{0}, \mathbf{e}_{1}, \ldots, \mathbf{e}_{d-1} \\
\sum_{i=1}^{2 i_{1}-i_{2}} \mathbf{e}_{i}+3 \sum_{i=2 i_{1}-i_{2}+1}^{d-1} \mathbf{e}_{i}+4 \mathbf{e}_{d}\end{array}$ \\
\hline$\Delta_{2}^{(4)}$ & $\begin{array}{l}i_{3} \leq i_{1}+i_{2} \\
i_{2}+i_{3}=d+1\end{array}$ & $2 \sum_{i=1}^{\mathbf{0}, \mathbf{e}_{1}, \ldots, \mathbf{e}_{d-1},} \mathbf{e}_{i}+\sum_{i=d-2 i_{1}+2, i \neq d}^{-2 i_{1}+1} \mathbf{e}_{i}+3 \sum_{i=-i_{1}+2 i_{2}+1}^{-i_{1}+2 i_{2}} \mathbf{e}_{i}+4 \mathbf{e}_{d}$ \\
\hline$\Delta_{3}^{(4)}$ & $\begin{array}{l}i_{3} \leq i_{1}+i_{2} \\
i_{1}+i_{2}+i_{3}=d+1\end{array}$ & $\underset{i=-i_{1}+i_{2}+i_{3}}{\mathbf{0}, \mathbf{e}_{1}, \ldots, \mathbf{e}_{d-2},} \mathbf{e}_{i}+2 \mathbf{e}_{d-1}, \quad \sum_{i=1}^{-i_{1}+i_{2}+i_{3}-1} \mathbf{e}_{i}+\sum_{i=2 i_{3}-1}^{d-2} \mathbf{e}_{i}+2 \mathbf{e}_{d}$ \\
\hline
\end{tabular}

TABLE 1 . The lattice simplices $\Delta \subset \mathbb{R}^{d}$ with $\operatorname{Vol}(\Delta) \leq 4$ in Theorem 1.2.

Second, the complete classification of the lattice spanning non-simplices $\mathcal{P} \subset \mathbb{R}^{d}$ with $\operatorname{Vol}(\mathcal{P}) \leq 4$ can be obtained from the following:

Theorem 1.3 ([12]). Let $2 \leq V \leq 4$ be a positive integer and $\mathcal{P} \subset \mathbb{R}^{d}$ a lattice spanning non-simplex with $\operatorname{Vol}(\mathcal{P})=V$. Assume that $\mathcal{P}$ is not a lattice pyramid. Then there exist up to unimodular equivalence exactly the following 24 possibilities for $\mathcal{P}$ :

(1) $\delta(\mathcal{P}, t)=1+t: \mathcal{P}^{(2)}$

(2) $\delta(\mathcal{P}, t)=1+2 t: \mathcal{P}_{i}^{(3)}, 1 \leq i \leq 2$;

(3) $\delta(\mathcal{P}, t)=1+t+t^{2}: \mathcal{Q}_{i}^{(3)}, 1 \leq i \leq 2$;

(4) $\delta(\mathcal{P}, t)=1+3 t: \mathcal{P}_{i}^{(4)}, 1 \leq i \leq 4$;

(5) $\delta(\mathcal{P}, t)=1+2 t+t^{2}: \mathcal{Q}_{i}^{(4)}, 1 \leq i \leq 9$;

(6) $\delta(\mathcal{P}, t)=1+t+2 t^{2}: \mathcal{R}_{i}^{(4)}, 1 \leq i \leq 2$; 
(7) $\delta(\mathcal{P}, t)=1+t+t^{2}+t^{3}: \mathcal{S}_{i}^{(4)}, 1 \leq i \leq 4 ;$

The dimension, vertices, and the $f$-vector of $\mathcal{P}$ are presented in TABLE 2 .

\begin{tabular}{|c|c|c|c|}
\hline & $d$ & vertices & $f$-vector \\
\hline $\mathcal{P}^{(2)}$ & 2 & $\mathbf{0}, \mathbf{e}_{1}, \mathbf{e}_{2}, \mathbf{e}_{1}+\mathbf{e}_{2}$ & $(1,4,4)$ \\
\hline $\mathcal{P}_{1}^{(3)}$ & 2 & $\mathbf{0}, 2 \mathbf{e}_{1}, \mathbf{e}_{2}, \mathbf{e}_{1}+\mathbf{e}_{2}$ & $(1,4,4)$ \\
\hline $\mathcal{P}_{2}^{(3)}$ & 3 & $\mathbf{0}, \mathbf{e}_{1}, \mathbf{e}_{2}, \mathbf{e}_{3}, \mathbf{e}_{1}+\mathbf{e}_{3}, \mathbf{e}_{2}+\mathbf{e}_{3}$ & $(1,6,9,5)$ \\
\hline $\mathcal{Q}_{1}^{(3)}$ & 3 & $\mathbf{0}, \mathbf{e}_{1}, \mathbf{e}_{2}, \mathbf{e}_{3}, \mathbf{e}_{1}+\mathbf{e}_{2}-2 \mathbf{e}_{3}$ & $(1,5,9,6)$ \\
\hline $\mathcal{Q}_{2}^{(3)}$ & 4 & $\mathbf{0}, \mathbf{e}_{1}, \mathbf{e}_{2}, \mathbf{e}_{3}, \mathbf{e}_{4},-\mathbf{e}_{1}-\mathbf{e}_{2}+\mathbf{e}_{3}+\mathbf{e}_{4}$ & $(1,6,15,18,9)$ \\
\hline $\mathcal{P}_{1}^{(4)}$ & 2 & $\mathbf{0}, 2 \mathbf{e}_{1}, \mathbf{e}_{2}, 2 \mathbf{e}_{1}+\mathbf{e}_{2}$ & $(1,4,4)$ \\
\hline $\mathcal{P}_{2}^{(4)}$ & 2 & $\mathbf{0}, 3 \mathbf{e}_{1}, \mathbf{e}_{1}+\mathbf{e}_{2}, 2 \mathbf{e}_{1}+\mathbf{e}_{2}$ & $(1,4,4)$ \\
\hline $\mathcal{P}_{3}^{(4)}$ & 3 & $\mathbf{0}, \mathbf{e}_{1}, \mathbf{e}_{2}, \mathbf{e}_{1}+\mathbf{e}_{3}, \mathbf{e}_{2}+\mathbf{e}_{3}, 2 \mathbf{e}_{3}$ & $(1,6,9,5)$ \\
\hline $\mathcal{P}_{4}^{(4)}$ & 4 & $\mathbf{0}, \mathbf{e}_{1}, \mathbf{e}_{2}, \mathbf{e}_{3}, \mathbf{e}_{4}, \mathbf{e}_{1}+\mathbf{e}_{2}, \mathbf{e}_{1}+\mathbf{e}_{3}, \mathbf{e}_{1}+\mathbf{e}_{4}$ & $(1,8,16,14,6)$ \\
\hline $\mathcal{Q}_{1}^{(4)}$ & 2 & $\mathbf{e}_{1},-\mathbf{e}_{2}, \mathbf{e}_{1}-\mathbf{e}_{2},-\mathbf{e}_{1}+\mathbf{e}_{2}$ & $(1,4,4)$ \\
\hline $\mathcal{Q}_{2}^{(4)}$ & 2 & $\mathbf{e}_{1}, \mathbf{e}_{2},-\mathbf{e}_{1},-\mathbf{e}_{2}$ & $(1,4,4)$ \\
\hline $\mathcal{Q}_{3}^{(4)}$ & 3 & $\mathbf{e}_{1}, \mathbf{e}_{2}, \mathbf{e}_{3}, \mathbf{e}_{1}+\mathbf{e}_{2},-\mathbf{e}_{3}$ & $(1,5,9,6)$ \\
\hline $\mathcal{Q}_{4}^{(4)}$ & 3 & $\mathbf{0}, \mathbf{e}_{1}, \mathbf{e}_{2}, \mathbf{e}_{1}+\mathbf{e}_{2}, 2 \mathbf{e}_{3}$ & $(1,5,8,5)$ \\
\hline $\mathcal{Q}_{5}^{(4)}$ & 3 & $\mathbf{0}, \mathbf{e}_{1}, \mathbf{e}_{2}, \mathbf{e}_{3}, \mathbf{e}_{1}+\mathbf{e}_{2}, \mathbf{e}_{1}+\mathbf{e}_{2}+\mathbf{e}_{3}$ & $(1,6,11,7)$ \\
\hline $\mathcal{Q}_{6}^{(4)}$ & 3 & $\mathbf{0}, \mathbf{e}_{1}, \mathbf{e}_{2}, \mathbf{e}_{3}, \mathbf{e}_{1}+\mathbf{e}_{2}, \mathbf{e}_{1}+\mathbf{e}_{2}-\mathbf{e}_{3}$ & $(1,6,12,8)$ \\
\hline $\mathcal{Q}_{7}^{(4)}$ & 4 & $\mathbf{0}, 2 \mathbf{e}_{1}, \mathbf{e}_{4}, \mathbf{e}_{2}+\mathbf{e}_{4}, \mathbf{e}_{3}+\mathbf{e}_{4}, \mathbf{e}_{2}+\mathbf{e}_{3}+\mathbf{e}_{4}$ & $(1,6,13,13,6)$ \\
\hline $\mathcal{Q}_{8}^{(4)}$ & 4 & $\mathbf{0}, \mathbf{e}_{1}, \mathbf{e}_{2}, \mathbf{e}_{1}+\mathbf{e}_{2}, \mathbf{e}_{3}, \mathbf{e}_{4}, \mathbf{e}_{3}+\mathbf{e}_{4}$ & $(1,7,17,18,8)$ \\
\hline $\mathcal{Q}_{9}^{(4)}$ & 5 & $\mathbf{0}, \mathbf{e}_{1}, \mathbf{e}_{2}, \mathbf{e}_{1}+\mathbf{e}_{2}, \mathbf{e}_{5}, \mathbf{e}_{3}+\mathbf{e}_{5}, \mathbf{e}_{4}+\mathbf{e}_{5}, \mathbf{e}_{3}+\mathbf{e}_{4}+\mathbf{e}_{5}$ & $(1,8,24,34,24,8)$ \\
\hline $\mathcal{R}_{1}^{(4)}$ & 3 & $\mathbf{0}, \mathbf{e}_{1}, \mathbf{e}_{2}, \mathbf{e}_{3}, \mathbf{e}_{1}+\mathbf{e}_{2}-3 \mathbf{e}_{3}$ & $(1,5,9,6)$ \\
\hline $\mathcal{R}_{2}^{(4)}$ & 4 & $\mathbf{0}, \mathbf{e}_{1}, \mathbf{e}_{2}, \mathbf{e}_{3}, \mathbf{e}_{4},-2 \mathbf{e}_{1}-\mathbf{e}_{2}+\mathbf{e}_{3}+\mathbf{e}_{4}$ & $(1,6,15,18,9)$ \\
\hline $\mathcal{S}_{1}^{(4)}$ & 4 & $\mathbf{0}, \mathbf{e}_{1}, \mathbf{e}_{2}, \mathbf{e}_{3}, \mathbf{e}_{4},-\mathbf{e}_{1}-\mathbf{e}_{2}-\mathbf{e}_{3}+\mathbf{e}_{4}$ & $(1,6,14,16,8)$ \\
\hline $\mathcal{S}_{2}^{(4)}$ & 4 & $\mathbf{0}, \mathbf{e}_{1}, \mathbf{e}_{2}, \mathbf{e}_{3}, \mathbf{e}_{4},-\mathbf{e}_{1}-\mathbf{e}_{2}-\mathbf{e}_{3}+2 \mathbf{e}_{4}$ & $(1,6,14,16,8)$ \\
\hline $\mathcal{S}_{3}^{(4)}$ & 5 & $\mathbf{0}, \mathbf{e}_{1}, \mathbf{e}_{2}, \mathbf{e}_{3}, \mathbf{e}_{4}, \mathbf{e}_{5},-2 \mathbf{e}_{1}-\mathbf{e}_{2}+\mathbf{e}_{3}+\mathbf{e}_{4}+\mathbf{e}_{5}$ & $(1,7,21,34,30,12)$ \\
\hline$\overline{\mathcal{S}_{4}^{(4)}}$ & 6 & $\mathbf{0}, \mathbf{e}_{1}, \mathbf{e}_{2}, \mathbf{e}_{3}, \mathbf{e}_{4}, \mathbf{e}_{5}, \mathbf{e}_{6},-\mathbf{e}_{1}-\mathbf{e}_{2}-\mathbf{e}_{3}+\mathbf{e}_{4}+\mathbf{e}_{5}+\mathbf{e}_{6}$ & $(1,8,28,56,68,48,16)$ \\
\hline
\end{tabular}

TABLE 2. The lattice spanning non-simplices $\mathcal{P}$ with $\operatorname{Vol}(\mathcal{P}) \leq 4$ in Theorem 1.3.

Finally, the complete classification of the lattice non-spanning non-simplices $\mathcal{P} \subset \mathbb{R}^{d}$ with $\operatorname{Vol}(\mathcal{P})=4$ can be obtained from the following:

Theorem 1.4. Let $\mathcal{P} \subset \mathbb{R}^{d}$ be a lattice non-spanning non-simplex with $\operatorname{Vol}(\mathcal{P})=4$. Assume that $\mathcal{P}$ is not a lattice pyramid. Then there exist, up to unimodular equivalence, exactly the following 4 possibilities for $\mathcal{P}$ :

(1) $\delta(\mathcal{P}, t)=1+t+t^{k}+t^{k+1}$ with $k \geq 2: \mathcal{A}_{i}^{(4)}, 1 \leq i \leq 3$; 
(2) $\delta(\mathcal{P}, t)=1+t+2 t^{k}$ with $k \geq 2: \mathcal{B}^{(4)}$.

The dimension and vertices of $\mathcal{P}$ are presented in TABLE 3. In particular, $\mathcal{P}$ is (non-unimodularly) equivalent to a pyramid over a square.

\begin{tabular}{l|l|l} 
& $d$ & vertices \\
\hline $\mathcal{A}_{1}^{(4)}$ & $2 k$ & $\mathbf{0}, \mathbf{e}_{1}, \ldots, \mathbf{e}_{d-1}, \sum_{j=2}^{d-1} \mathbf{e}_{j}+2 \mathbf{e}_{d},-\mathbf{e}_{1}+\mathbf{e}_{2}$ \\
\hline $\mathcal{A}_{2}^{(4)}$ & $2 k+1$ & $\mathbf{0}, \mathbf{e}_{1}, \ldots, \mathbf{e}_{d-1}, \sum_{j=3}^{d-1} \mathbf{e}_{j}+2 \mathbf{e}_{d}, \mathbf{e}_{1}+\mathbf{e}_{2}$ \\
\hline $\mathcal{A}_{3}^{(4)}$ & $2 k+2$ & $\mathbf{0}, \mathbf{e}_{1}, \ldots, \mathbf{e}_{d-1}, \sum_{j=4}^{d-1} \mathbf{e}_{j}+2 \mathbf{e}_{d}, \mathbf{e}_{1}+\mathbf{e}_{2}-\mathbf{e}_{3}$ \\
\hline $\mathcal{B}^{(4)}$ & $2 k$ & $\mathbf{0}, \mathbf{e}_{1}, \ldots, \mathbf{e}_{d-1}, \sum_{j=2}^{d-1} \mathbf{e}_{j}+2 \mathbf{e}_{d}, \mathbf{e}_{1}-\mathbf{e}_{2}$
\end{tabular}

TABLE 3. The lattice non-spanning non-simplices $\mathcal{P}$ with $\operatorname{Vol}(\mathcal{P})=4$ in Theorem 1.4, where $k \geq 2$.

The present paper is organized as follows: First, in Section 2, we introduce basic materials on lattice polytopes and summarize lemmata which will be indispensable in what follows. We then, in Section 3, prove Theorem 1.2. Finally, in Section 4, we prove Theorem 1.4.

Acknowledgment. The authors would like to thank Gabriele Balletti for pointing out minor imperfections in our classification done in our first draft and, in addition, for informing them about the paper [12]. The authors would like to thank anonymous referees for their careful readings and many helpful comments which make this paper more readable, in particular, the proof of Theorem 1.4 was significantly shortened. The first author is partially supported by JSPS KAKENHI 19H00637. The second author is partially supported by Grant-in-Aid for JSPS Fellows 16J01549.

\section{BASIC MATERIALS ON LATTICE POLYTOPES}

In this section, we recall basic materials on lattice polytopes and we prepare essential lemmata in this paper. First, we introduce the associated finite abelian groups of lattice simplices. For a lattice simplex $\Delta \subset \mathbb{R}^{d}$ of dimension $d$ whose vertices are $\mathbf{v}_{0}, \ldots, \mathbf{v}_{d} \in \mathbb{Z}^{d}$, set

$$
\Lambda_{\Delta}=\left\{\left(\lambda_{0}, \ldots, \lambda_{d}\right) \in(\mathbb{R} / \mathbb{Z})^{d+1}: \sum_{i=0}^{d} \lambda_{i}\left(\mathbf{v}_{i}, 1\right) \in \mathbb{Z}^{d+1}\right\} .
$$

The collection $\Lambda_{\Delta}$ forms a finite abelian group with addition defined as follows: For $\left(\lambda_{0}, \ldots, \lambda_{d}\right) \in(\mathbb{R} / \mathbb{Z})^{d+1}$ and $\left(\lambda_{0}^{\prime}, \ldots, \lambda_{d}^{\prime}\right) \in(\mathbb{R} / \mathbb{Z})^{d+1},\left(\lambda_{0}, \ldots, \lambda_{d}\right)+$ $\left(\lambda_{0}^{\prime}, \ldots, \lambda_{d}^{\prime}\right)=\left(\lambda_{0}+\lambda_{0}^{\prime}, \ldots, \lambda_{d}+\lambda_{d}^{\prime}\right) \in(\mathbb{R} / \mathbb{Z})^{d+1}$. We denote the unit of $\Lambda_{\Delta}$ by $\mathbf{0}$, and the inverse of $\lambda$ by $-\lambda$, and also denote $\underbrace{\lambda+\cdots+\lambda}_{j}$ by $j \lambda$

for an integer $j>0$ and $\lambda \in \Lambda_{\Delta}$. For $\lambda=\left(\lambda_{0}, \ldots, \lambda_{d}\right) \in \Lambda_{\Delta}$, where each $\lambda_{i}$ is taken with $0 \leq \lambda_{i}<1$, we set $\operatorname{ht}(\lambda)=\sum_{i=0}^{d} \lambda_{i} \in \mathbb{Z}$, and $\operatorname{ord}(\lambda)=\min \left\{\ell \in \mathbb{Z}_{>0}: \ell \lambda=\mathbf{0}\right\}$. 
In [4], it is shown that there is a bijection between unimodular equivalence classes of $d$-dimensional lattice simplices with a chosen ordering of their vertices and finite abelian subgroups of $(\mathbb{R} / \mathbb{Z})^{d+1}$ such that the sum of all entries of each element is an integer. In particular, two lattice simplices $\Delta$ and $\Delta^{\prime}$ are unimodularly equivalent if and only if there exist orderings of their vertices such that $\Lambda_{\Delta}=\Lambda_{\Delta^{\prime}}$. Moreover, we can characterize lattice pyramids in terms of the associated finite abelian groups by using the following lemma.

Lemma 2.1 ([22, Lemma 12]). Let $\Delta \subset \mathbb{R}^{d}$ be a lattice simplex of dimension $d$. Then $\Delta$ is a lattice pyramid if and only if there is $i \in\{0, \ldots, d\}$ such that $\lambda_{i}=0$ for all $\left(\lambda_{0}, \ldots, \lambda_{d}\right) \in \Lambda_{\Delta}$.

It is well known that the $\delta$-polynomial of the lattice simplex $\Delta$ can be computed as follows:

Lemma 2.2 ([6, Proposition 2.6]). Let $\Delta$ be a lattice simplex of dimension $d$ whose $\delta$-polynomial equals $\delta_{0}+\delta_{1} t+\cdots+\delta_{d} t^{d}$. Then for each $i$, we have $\delta_{i}=\left|\left\{\lambda \in \Lambda_{\Delta}: \operatorname{ht}(\lambda)=i\right\}\right|$. In particular, one has $\operatorname{Vol}(\Delta)=\left|\Lambda_{\Delta}\right|$.

Let $\mathcal{P} \subset \mathbb{R}^{d}$ be a lattice polytope of dimension $d$. Given integers $n=$ $1,2, \ldots$, we define the function $L_{\mathcal{P}}(n)$ as follows:

$$
L_{\mathcal{P}}(n):=\left|n \mathcal{P} \cap \mathbb{Z}^{d}\right| \text {. }
$$

Then it is known that $L_{\mathcal{P}}(n)$ is a polynomial in $n$ of degree $d$ with $L_{\mathcal{P}}(0)=$ 1 (see [11]). We call $L_{\mathcal{P}}(n)$ the Ehrhart polynomial of $\mathcal{P}$. The Ehrhart polynomial $L_{\mathcal{P}}(n)$ can be computed by using the $\delta$-vector of $\mathcal{P}$.

Lemma 2.3. Let $\mathcal{P} \subset \mathbb{R}^{d}$ be a lattice polytope of dimension $d$ and $\left(\delta_{0}, \ldots, \delta_{d}\right)$ the $\delta$-vector of $\mathcal{P}$. Then one has

$$
L_{\mathcal{P}}(n)=\sum_{i=0}^{d} \delta_{i}\left(\begin{array}{c}
n+d-i \\
d
\end{array}\right) .
$$

Finally, we recall a useful technique to compute Ehrhart polynomials.

Lemma 2.4. Let $\mathcal{P} \subset \mathbb{R}^{d}$ be a lattice non-simplex. Assume that $\mathcal{P}$ has a lattice triangulation which consists of two maximal simplices $\Delta_{1}$ and $\Delta_{2}$. Then one has $L_{\mathcal{P}}(n)=L_{\Delta_{1}}(n)+L_{\Delta_{2}}(n)-L_{\Delta_{1} \cap \Delta_{2}}(n)$.

\section{Proof of Theorem 1.2}

In this section, we classify the lattice simplices $\Delta \subset \mathbb{R}^{d}$ of dimension $d$ with $\operatorname{Vol}(\Delta) \leq 4$ up to unimodular equivalence and lattice pyramid constructions. Namely, we prove Theorem 1.2. In order to do this job, we consider the following three cases:

(1) $\operatorname{Vol}(\Delta)=2($ Subsection 3.1$)$;

(2) $\operatorname{Vol}(\Delta)=3$ (Subsection 3.2);

(3) $\operatorname{Vol}(\Delta)=4$ (Subsection 3.3). 
3.1. The case $\operatorname{Vol}(\Delta)=2$. In this subsection, we consider the case where $\operatorname{Vol}(\Delta)=2$. It follows from Theorem 1.1 (1) that $i_{1} \leq\lfloor(d+1) / 2\rfloor$. Since $\left|\Lambda_{\Delta}\right|=2$, for any $\lambda \in \Lambda_{\Delta} \backslash\{\mathbf{0}\}, \operatorname{ord}(\lambda)=2$. Hence since $\Delta$ is not a lattice pyramid, by using Lemma 2.1 , it follows that $\Lambda_{\Delta}$ is generated by one element $(1 / 2, \ldots, 1 / 2)$ and $d+1$ is an even number. By using Lemma 2.2, one has $2 i_{1}=d+1$. Moreover it is easy to see that $\Lambda_{\Delta(2)}=\Lambda_{\Delta}$ with any ordering of the vertices of $\Delta^{(2)}$. Hence this completes the proof of the case where $\operatorname{Vol}(\Delta)=2$.

3.2. The case $\operatorname{Vol}(\Delta)=3$. In this subsection, we consider the case where $\operatorname{Vol}(\Delta)=3$. It follows from Theorem $1.1(2)$ that $2 i_{1} \geq i_{2}$ and $i_{1}+i_{2}=$ $(d+1) / 2$. For nonnegative integers $a$ and $b$, we let $\Lambda(a, b)$ be the finite abelian subgroups of $(\mathbb{R} / \mathbb{Z})^{a+b}$ defined as follows:

$$
\Lambda(a, b)=\left\langle(\underbrace{\frac{1}{3}, \ldots, \frac{1}{3}}_{a}, \underbrace{\frac{2}{3}, \ldots, \frac{2}{3}}_{b})\right\rangle \text {. }
$$

Since $\operatorname{Vol}(\Delta)=\left|\Lambda_{\Delta}\right|=3$, for any $\lambda \in \Lambda_{\Delta} \backslash\{\mathbf{0}\}, \operatorname{ord}(\lambda)=3$. Hence since $\Delta$ is not a lattice pyramid, by Lemma 2.1 , there exist nonnegative integers $a, b$ with $a+b=d+1$ such that $\Lambda_{\Delta}=\Lambda(a, b)$ with some ordering of the vertices of $\Delta$. Since $\Lambda(a, b)$ coincides with $\Lambda(b, a)$ by reordering of the coordinates, we can assume that $a \geq b$. Then by using Lemma 2.2 , one has $i_{1}=(a+2 b) / 3$ and $i_{2}=(2 a+b) / 3$. Hence we obtain $a=-i_{1}+2 i_{2}, b=2 i_{1}-i_{2}$ and $d+1=a+b=i_{1}+i_{2}$. Moreover, it is easy to see that $\Lambda_{\Delta^{(3)}}=\Lambda(a, b)$ with some ordering of the vertices of $\Delta^{(3)}$. Hence this completes the proof of the case where $\operatorname{Vol}(\Delta)=3$.

3.3. The case $\operatorname{Vol}(\Delta)=4$. In this subsection, we consider the case where $\operatorname{Vol}(\Delta)=4$. For nonnegative integers $a, b, c$, we let $\Lambda_{1}(a, b, c)$ and $\Lambda_{2}(a, b, c)$ be the finite abelian subgroups of $(\mathbb{R} / \mathbb{Z})^{a+b+c}$ defined as follows:

$$
\Lambda_{1}(a, b, c)=\left\langle(\underbrace{\frac{1}{4}, \ldots, \frac{1}{4}}_{a}, \underbrace{\frac{1}{2}, \ldots, \frac{1}{2}}_{b}, \underbrace{\frac{3}{4}, \ldots, \frac{3}{4}}_{c})\right\rangle
$$

$\Lambda_{2}(a, b, c)=\left\langle(\underbrace{\frac{1}{2}, \ldots, \frac{1}{2}}_{a}, \underbrace{\frac{1}{2}, \ldots, \frac{1}{2}}_{b}, \underbrace{0, \ldots, 0}_{c}),(\underbrace{0, \ldots, 0}_{a}, \underbrace{\frac{1}{2}, \ldots, \frac{1}{2},}_{b} \underbrace{\frac{1}{2}, \ldots, \frac{1}{2}}_{c})\right\rangle$.

Since $\operatorname{Vol}(\Delta)=\left|\Lambda_{\Delta}\right|=4$, for any $\lambda \in \Lambda_{\Delta} \backslash\{\mathbf{0}\}$, $\operatorname{ord}(\lambda) \in\{2,4\}$. Hence since $\Delta$ is not a lattice pyramid, by Lemma 2.1 , there exist nonnegative integers $a, b, c$ with $d+1=a+b+c$ such that $\Lambda_{\Delta}$ coincides with $\Lambda_{1}(a, b, c)$ or $\Lambda_{2}(a, b, c)$ with some ordering of the vertices of $\Delta$.

First, suppose that $\Lambda_{\Delta}=\Lambda_{1}(a, b, c)$ with some ordering of the vertices of $\Delta$. Then since $\Lambda_{1}(a, b, c)$ coincides with $\Lambda_{1}(c, b, a)$ by reordering of the 
coordinates, we may assume that $a \geq c$. Moreover, by using Lemma 2.2, one has $\left\{i_{1}, i_{2}, i_{3}\right\}=\{(a+2 b+3 c) / 4,(a+c) / 2,(3 a+2 b+c) / 4\}$. Set $\left(h_{1}, h_{2}, h_{3}\right)=$ $((a+2 b+3 c) / 4,(a+c) / 2,(3 a+2 b+c) / 4)$. Then we obtain $a=-h_{1}+h_{2}+$ $h_{3}, b=h_{1}-2 h_{2}+h_{3}$ and $c=h_{1}+h_{2}-h_{3}$. Since $a \geq c,\left(h_{1}, h_{3}\right)$ is $\left(i_{1}, i_{2}\right)$, $\left(i_{1}, i_{3}\right)$ or $\left(i_{2}, i_{3}\right)$. Hence it follows from $a, b, c \geq 0$ that one of the following conditions is satisfied:

(1) $i_{1}+i_{3} \geq 2 i_{2}$ and $d+1=i_{1}+i_{3}$, and $\Lambda_{\Delta}=\Lambda_{1}\left(-i_{1}+i_{2}+i_{3}, i_{1}-\right.$ $\left.2 i_{2}+i_{3}, i_{1}+i_{2}-i_{3}\right)$

(2) $i_{2}+i_{3} \geq 2 i_{1}$ and $d+1=i_{2}+i_{3}$, and $\Lambda_{\Delta}=\Lambda_{1}\left(i_{1}-i_{2}+i_{3},-2 i_{1}+\right.$ $\left.i_{2}+i_{3}, i_{1}+i_{2}-i_{3}\right)$

(3) $i_{1}+i_{2} \geq 2 i_{3}$ and $d+1=i_{1}+i_{2}$, and $\Lambda_{\Delta}=\Lambda_{1}\left(-i_{1}+i_{2}+i_{3}, i_{1}+\right.$ $\left.i_{2}-2 i_{3}, i_{1}-i_{2}+i_{3}\right)$;

If $i_{1}=i_{2}$ or $i_{2}=i_{3}$, then the condition (1) is equivalent to one of the conditions (2) and (3). Since $i_{1}+i_{2} \geq 2 i_{3}$ implies that $i_{1}=i_{2}=i_{3}$, if the condition (3) is satisfied, then condition (2) is satisfied. Moreover, it always follows that $i_{2}+i_{3} \geq 2 i_{1}$. Hence we know that one of the following conditions is satisfied:

(1') $i_{1}<i_{2}<i_{3}, i_{1}+i_{3} \geq 2 i_{2}$ and $d+1=i_{1}+i_{3}$, and $\Lambda_{\Delta}=\Lambda_{1}\left(-i_{1}+\right.$ $\left.i_{2}+i_{3}, i_{1}-2 i_{2}+i_{3}, i_{1}+i_{2}-i_{3}\right)$

(2') $d+1=i_{2}+i_{3}$ and $\Lambda_{\Delta}=\Lambda_{1}\left(i_{1}-i_{2}+i_{3},-2 i_{1}+i_{2}+i_{3}, i_{1}+i_{2}-i_{3}\right)$.

Now, we assume that the condition $\left(1^{\prime}\right)$ is satisfied. Then the conditions $i_{1}+i_{3} \leq 2 i_{2}, i_{2} \leq\lfloor(d+1) / 2\rfloor$ and $i_{1}+i_{3}=d+1$ imply

$$
2 i_{2}=i_{1}+i_{3}=d+1 \text {. }
$$

This condition and the conditions $i_{1}<i_{2}<i_{3}$ and $i_{3} \leq i_{1}+i_{2}$ satisfy the condition of Theorem 1.1 (3). Moreover, then, it is easy to see that $\Lambda_{\Delta_{1}^{(4)}}=\Lambda_{1}(a, b, c)$ with some ordering of the vertices of $\Delta_{1}^{(4)}$. On the other hand, we assume that the condition (2') is satisfied. Then the conditions $i_{3} \leq i_{1}+i_{2}$ and $i_{2}+i_{3}=d+1$ satisfy the condition of Theorem 1.1 (3). Moreover, then, it is easy to see that $\Lambda_{\Delta_{2}^{(4)}}=\Lambda_{1}(a, b, c)$ with some ordering of the vertices of $\Delta_{2}^{(4)}$.

Next, we suppose that $\Lambda_{\Delta}=\Lambda_{2}(a, b, c)$ with some ordering of the vertices of $\Delta$. It follows that $\Lambda_{2}(a, b, c)$ coincides with $\Lambda_{2}(b, a, c)\left(\right.$ resp. $\left.\Lambda_{2}(c, b, a)\right)$ by reordering of the coordinates. Hence we may assume that $a \geq b \geq c$. Then by using Lemma 2.2 , one has $\left(i_{1}, i_{2}, i_{3}\right)=((b+c) / 2,(a+c) / 2,(a+b) / 2)$. Therefore, we obtain $d+1=i_{1}+i_{2}+i_{3}$ and $\Lambda_{\Delta}=\Lambda_{2}\left(-i_{1}+i_{2}+i_{3}, i_{1}-\right.$ $\left.i_{2}+i_{3}, i_{1}+i_{2}-i_{3}\right)$. The conditions $i_{3} \leq i_{1}+i_{2}$ and $i_{1}+i_{2}+i_{3}=d+1$ satisfy the condition of Theorem 1.1 (3). Moreover, it is easy to see that $\Lambda_{\Delta_{3}^{(4)}}=\Lambda_{2}(a, b, c)$ with some ordering of the vertices of $\Delta_{3}^{(4)}$. Hence this completes the proof of the case where $\operatorname{Vol}(\Delta)=4$.

Therefore, Theorem 1.2 follows. 


\section{Proof of Theorem 1.4}

In this section, we prove Theorem 1.4. First, we show the following.

Lemma 4.1. One has $\delta\left(\mathcal{A}_{i}^{(4)}, t\right)=1+t+t^{k}+t^{k+1}$ for $1 \leq i \leq 3$ and $\delta\left(\mathcal{B}^{(4)}, t\right)=1+t+2 t^{k}$.

Proof. We show that $\delta\left(\mathcal{B}^{(4)}, t\right)=1+t+2 t^{k}$. Let $\Delta_{1}=\operatorname{conv}\left(\mathbf{0}, \mathbf{e}_{1}, \ldots, \mathbf{e}_{d-1}, \mathbf{e}_{2}+\right.$ $\left.\cdots+\mathbf{e}_{d-1}+2 \mathbf{e}_{d}\right)$ and $\Delta_{2}=\operatorname{conv}\left(\mathbf{e}_{1}, \ldots, \mathbf{e}_{d-1}, \mathbf{e}_{2}+\cdots+\mathbf{e}_{d-1}+2 \mathbf{e}_{d}, \mathbf{e}_{1}+\mathbf{e}_{2}\right)$. Then $\mathcal{B}^{(4)}$ has a lattice triangulation which consists of two maximal simplices $\Delta_{1}$ and $\Delta_{2}$. Indeed, the hyperplane

$$
\mathcal{H}=\left\{\left(x_{1}, \ldots, x_{d}\right) \in \mathbb{R}^{d}: 2\left(x_{1}+\cdots+x_{d-1}\right)-(2 k-1) x_{d}=2\right\}
$$

divides $\mathcal{B}^{(4)}$ into $\Delta_{1}$ and $\Delta_{2}$. Moreover, one has $\delta\left(\Delta_{1}, t\right)=\delta\left(\Delta_{2}, t\right)=1+t^{k}$ and $\delta\left(\Delta_{1} \cap \Delta_{2}, t\right)=1$. Hence by using Lemmas 2.3 and 2.4, we obtain

$$
\begin{aligned}
L_{\mathcal{B}^{(4)}}(n) & =\left(\begin{array}{c}
n+d \\
d
\end{array}\right)+2\left(\begin{array}{c}
n+d-k \\
d
\end{array}\right)+\left(\left(\begin{array}{c}
n+d \\
d
\end{array}\right)-\left(\begin{array}{c}
n+d-1 \\
d-1
\end{array}\right)\right) \\
& =\left(\begin{array}{c}
n+d \\
d
\end{array}\right)+2\left(\begin{array}{c}
n+d-k \\
d
\end{array}\right)+\left(\begin{array}{c}
n+d-1 \\
d
\end{array}\right) .
\end{aligned}
$$

Therefore, one has $\delta\left(\mathcal{B}^{(4)}, t\right)=1+t+2 t^{k}$. The remaining cases can be shown by the same way.

Let $\mathcal{P} \subset \mathbb{R}^{d}$ be a lattice non-spanning non-simplex of dimension $d$ with $\operatorname{Vol}(\mathcal{P})=4$. Assume that $\mathcal{P}$ is not a lattice pyramid. Finally, we show that $\mathcal{P}$ is unimodularly equivalent to one of the lattice polytopes in Theorem 1.4. We consider the affine lattice $L_{\mathcal{P}}$ generated by $\mathcal{P} \cap \mathbb{Z}^{d}$. Let $\tilde{\mathcal{P}} \subset \mathbb{R}^{d}$ be the lattice spanning polytope given by the vertices of $\mathcal{P}$ with respect to the lattice $L_{\mathcal{P}}$. Since $\mathcal{P}$ is not a simplex and is not spanning, the normalized volume of $\tilde{\mathcal{P}}$ is equal to 2 . Hence by Theorem $1.3, \tilde{\mathcal{P}}$ is unimodularly equivalent to a lattice pyramid of a unit square. In particular, four vertices $\tilde{\mathbf{v}}_{1}, \ldots, \tilde{\mathbf{v}}_{4}$ of $\tilde{\mathcal{P}}$ satisfy the relation $\tilde{\mathbf{v}}_{1}+\tilde{\mathbf{v}}_{2}=\tilde{\mathbf{v}}_{3}+\tilde{\mathbf{v}}_{4}$. Therefore, $\mathcal{P}$ can be determined as $\mathcal{P}=\operatorname{conv}(S \cup\{\mathbf{u}\})$, for an empty simplex $S$ of normalized volume 2 , and a lattice point $u=\left(u_{1}, \ldots, u_{d}\right)$ satisfying $\mathbf{u}=\mathbf{v}_{1}+\mathbf{v}_{2}-\mathbf{v}_{3}$, for some $\mathbf{v}_{i}$ chosen among the vertices of $S$. By Theorem 1.2, we can assume that

$$
S=\operatorname{conv}\left(\mathbf{0}, \mathbf{e}_{1}, \ldots, \mathbf{e}_{d-1}, \mathbf{e}_{a+1}+\cdots+\mathbf{e}_{d-1}+2 \mathbf{e}_{d}\right)
$$

for some integer $a \geq 0$. If $a \geq 4$, then for some $1 \leq i \leq a, u_{i}=0$. This implies that $\mathcal{P}$ is a lattice pyramid. Hence one has $a \leq 3$.

4.1. The case $a=3$. If $d$ is odd, then $S$ is unimodularly equivalent to

$$
\operatorname{conv}\left(\mathbf{0}, \mathbf{e}_{1}, \ldots, \mathbf{e}_{d-1}, \mathbf{e}_{a+2}+\cdots+\mathbf{e}_{d-1}+2 \mathbf{e}_{d}\right) .
$$

Hence we can assume that $d$ is even. Since $\mathcal{P}$ is not a lattice pyramid, we obtain $\left\{\mathbf{v}_{1}, \mathbf{v}_{2}, \mathbf{v}_{3}\right\}=\left\{\mathbf{e}_{1}, \mathbf{e}_{2}, \mathbf{e}_{3}\right\}$. Therefore, we may suppose that $\mathbf{u}=$ $(1,1,-1,0, \ldots, 0)$. Thus $\mathcal{P}=\mathcal{A}_{3}^{(4)}$. 
4.2. The case $a=2$. Similarly, we can assume that $d$ is odd. Moreover, since $\mathcal{P}$ is not a lattice pyramid, we obtain $\left\{\mathbf{v}_{1}, \mathbf{v}_{2}, \mathbf{v}_{3}\right\} \supset\left\{\mathbf{e}_{1}, \mathbf{e}_{2}\right\}$. Hence we may suppose that $\mathbf{u}=\mathbf{e}_{1}+\mathbf{e}_{2}-\mathbf{v}$ or $\mathbf{e}_{1}-\mathbf{e}_{2}+\mathbf{v}$, where $\mathbf{v} \in\left\{\mathbf{0}, \mathbf{e}_{3}, \mathbf{e}_{3}+\right.$ $\left.\cdots+\mathbf{e}_{d-1}+2 \mathbf{e}_{d}\right\}$. For any case, $\mathcal{P}$ is unimodularly equivalent to $\mathcal{A}_{2}^{(4)}$.

4.3. The case $a=1$. Similarly, we can assume that $d$ is even. Moreover, since $\mathcal{P}$ is not a lattice pyramid, we obtain $\mathbf{e}_{1} \in\left\{\mathbf{v}_{1}, \mathbf{v}_{2}, \mathbf{v}_{3}\right\}$. Hence we may suppose that $\mathbf{u}=\mathbf{e}_{1}+\mathbf{u}_{1}-\mathbf{u}_{2}$ or $-\mathbf{e}_{1}+\mathbf{u}_{1}+\mathbf{u}_{2}$, where $\mathbf{u}_{1}, \mathbf{u}_{2} \in$ $\left\{\mathbf{0}, \mathbf{e}_{2}, \mathbf{e}_{3}, \mathbf{e}_{2}+\cdots+\mathbf{e}_{d-1}+2 \mathbf{e}_{d}\right\}$. When $\mathbf{u}=\mathbf{e}_{1}+\mathbf{u}_{1}-\mathbf{u}_{2}, \mathcal{P}$ is unimodularly equivalent to $\mathcal{B}^{(4)}$. When $\mathbf{u}=-\mathbf{e}_{1}+\mathbf{u}_{1}+\mathbf{u}_{2}, \mathcal{P}$ is unimodularly equivalent to $\mathcal{A}_{1}^{(4)}$.

4.4. The case $a=0$. Similarly, we can assume that $d$ is odd. Moreover, we may suppose that $\mathbf{e}_{1} \in\left\{\mathbf{v}_{1}, \mathbf{v}_{2}, \mathbf{v}_{3}\right\}$. When $\mathbf{v}_{1}=\mathbf{e}_{1}$, we should consider $\left(\mathbf{v}_{2}, \mathbf{v}_{3}\right)=\left(\mathbf{e}_{2}, \mathbf{e}_{3}\right)$ or $\left\{\mathbf{v}_{2}, \mathbf{v}_{3}\right\} \subset\left\{\mathbf{0}, \mathbf{e}_{1}, \mathbf{e}_{1}+\cdots+\mathbf{e}_{d-1}+2 \mathbf{e}_{d}\right\}$. For each case,

$\mathcal{P}$ is unimodularly equivalent to $\mathcal{A}_{2}^{(4)}$. When $\mathbf{v}_{3}=\mathbf{e}_{1}$, we should consider only the case $\left(\mathbf{v}_{1}, \mathbf{v}_{2}\right)=\left(\mathbf{0}, \mathbf{e}_{1}+\cdots+\mathbf{e}_{d-1}+2 \mathbf{e}_{d}\right)$. In this case, $\mathcal{P}$ is also unimodularly equivalent to $\mathcal{A}_{2}^{(4)}$.

Therefore, we complete the proof of Theorem 1.4.

\section{REFERENCES}

[1] G. Balletti and A. M. Kasprzyk, Three-dimensional lattice polytopes with two interior lattice points, arXiv:1612.08918.

[2] V. V. Batyrev, Lattice polytopes with a given $h^{*}$-polynomial, Algebraic and geometric combinatorics, Contemp. Math., vol. 423, Amer. Math. Soc., Providence, RI, 2006, $1-10$.

[3] V.V. Batyrev and J. Hofscheier, A generalization of a theorem of G. K. White, arXiv:1004.3411.

[4] V.V. Batyrev and J. Hofscheier, Lattice polytopes, finite abelian subgroups in $\mathrm{SL}(n, \mathbb{C})$ and coding theory, arXiv:1309.5312.

[5] V. Batyrev and D. Juny, Classification of Gorenstein toric del Pezzo varieties in arbitrary dimension, Mosc. Math. J., 10(2010), 285-316.

[6] V. Batyrev and B. Nill, Multiples of lattice polytopes without interior lattice points, Mosc. Math. J., 7(2007), 195-207.

[7] M. Beck and S. Robins, "Computing the continuous discretely", Undergraduate Texts in Mathematics, Springer, second edition, 2015.

[8] M. Blanco and F. Santos, Lattice 3-polytopes with few lattice points. SIAM J. Discrete Math., 30(2016), 669-686.

[9] M. Blanco and F. Santos, Lattice 3-polytopes with 6 lattice points, SIAM J. Discrete Math., 30(2016), 687-717.

[10] M. Blanco and F. Santos, Enumeration of Lattice 3-Polytopes by Their Number of Lattice Points, Discrete Comput. Geom., 60(2018), 756-800.

[11] E. Ehrhart, "Polynomês Arithmétiques et Méthode des Polyédres en Combinatorie", Birkhäuser, Boston/Basel/Stuttgart, 1977.

[12] A. Esterov and G. Gusev, Multivariate Abel-Ruffini, Math. Ann., 365(2016), 10911110.

[13] G. Ewald, On the classification of toric Fano varieties, Disc. Comput. Geom. 3 (1988), 49-54. 
[14] T. Hibi, "Algebraic Combinatorics on Convex Polytopes," Carslaw Publications, Glebe NSW, Australia, 1992.

[15] T. Hibi, A lower bound theorem for Ehrhart polynomials of convex polytopes, $A d v$. Math., 105(1994), 162-165.

[16] T. Hibi, A. Higashitani and N. Li, Hermite normal forms of $\delta$-vectors, J. Combin. Theory Ser. A 119(2012), 1158-1173.

[17] T. Hibi, A. Higashitani and Y. Nagazawa, Ehrhart polynomials of convex polytopes with small volumes, Euro. J. Combin. 32(2011), 226-232.

[18] A. Higashitani, Ehrhart polynomials of integral simplices with prime volumes, INTEGERS, 14(2014), 1-15.

[19] A. Higashitani, B. Nill and A. Tsuchiya, Gorenstein polytopes with trinomial $h^{*}$ polynomials, arXiv:1503.05685.

[20] J. Hofscheier, L. Katthän and B. Nill, Ehrhart Theory of Spanning Lattice Polytopes, International Mathematics Research Notices, 2018 (2018), 5947-5973.

[21] A. M. Kasprzyk, Canonical toric Fano threefolds, Canad. J. Math., 62(2010),12931309.

[22] B. Nill, Lattice polytopes having $h^{*}$-polynomials with given degree and linear coefficient, European J. Combin., 29, 1596-1602, 2008.

[23] R. P. Stanley, Decompositions of rational convex polytopes, Annals of Discrete Math. 6(1980), 333-342.

[24] R. P. Stanley. Enumerative combinatorics. Volume 1, volume 49 of Cambridge Studies in Advanced Mathematics. Cambridge University Press, Cambridge, second edition, 2012.

[25] A. Tsuchiya, Ehrhart polynomials of lattice polytopes with normalized volumes $5, J$. Comb., 10 (2019), 283-290.

[26] V. E. Voskresenskii and A. A. Klyachko, Toroidal Fano varieties and root system, Math. USSR Izvestiya 24(1985), 221-244.

(Takayuki Hibi) Department of Pure and Applied Mathematics, Graduate School of Information Science and Technology, Osaka University, Suita, OsAKA 565-0871, JAPAN

E-mail address: hibi@math.sci.osaka-u.ac.jp

(Akiyoshi Tsuchiya) Graduate school of Mathematical Sciences, University of Tokyo, Komaba, Meguro-Ku, Tokyo 153-8914, Japan

E-mail address: akiyoshi@ms.u-tokyo.ac.jp 